Aim of the study: To determine the significance of expression of synaptophysin, chromogranin $\mathrm{A}$, and $\mathrm{Ki}-67$ and their association with clinicopathological parameters, and to find out the possible prognostic factors in gastric neuroendocrine carcinoma (G-NEC).

Material and methods: We investigated the immunohistochemical features and prognosis of 62 G-NECs, and evaluated the association among expressions of synaptophysin, chromogra$\operatorname{nin} \mathrm{A}$, and $\mathrm{Ki}-67$, clinicopathological variables, and outcome.

Results: Chromogranin A expression was found more commonly in smallcell NECs $(9 / 9,100 \%)$ than in largecell NECs $(27 / 53,51 \%)(p=0.008)$. No statistical significance was found in $\mathrm{Ki}-67(p=0.494)$ or synaptophysin $(p>0.1)$ expression between NEC cell types. Correlation analyses revealed that Ki-67 expression was significantly associated with mid-third disease of stomach $(p=0.005)$ and vascular involvement $(p=0.006)$, and had a trend of significant correlation with tumour relapse $(p=0.078)$. High expression of chromogranin A was significantly associated with histology of small-cell NECs $(p=0.008)$ and lesser tumour greatest dimension $(p=0.038)$. The prognostic significance was determined by means of Kaplan-Meier survival estimates and log-rank tests, and as a result, early TNM staging and postoperative chemotherapy were found to be correlated with longer overall survival $(p<0.05)$. Univariate analysis revealed associations between poor prognosis in NECs and several factors, including high TNM staging ( $p=0.048)$, vascular involvement $(p=0.023)$, relapse $(p=0.004)$, and microscopic/macroscopic residual tumour (R1/2, $p<0.001)$. In a multivariate analysis, relapse was identified as the sole independent prognostic factor.

Conclusions: No significant correlation between survival and expression of synaptophysin, chromogranin A, or $\mathrm{Ki}-67$ has been determined in G-NECs. Our study indicated that early diagnosis, no-residual-tumour resection, and postoperative chemotherapy were possible prognostic factors.

Key words: neuroendocrine carcinoma, stomach, prognosis, Ki-67, chromogranin A.

Contemp Oncol (Pozn) 2016; 20 (4): 311-319 DOI: $10.5114 /$ wo. 2016.61852

\section{Histological characterisation and prognostic evaluation of 62 gastric neuroendocrine carcinomas}

\author{
Yujie Deng ${ }^{1^{*}}$, Xiaohui Chen ${ }^{2^{*}}$, Yuhong Ye ${ }^{3}, \mathrm{Xi} \mathrm{Shi}^{1}$, Kunshou Zhu ${ }^{2}$, \\ Liming Huang ${ }^{1}$, Sheng Zhang ${ }^{3}$, Mingang Ying ${ }^{2}$, Xuede Lin $^{1}$
}

\author{
'Department of Chemotherapy, The First Affiliated Hospital of Fujian Medical University, \\ Fuzhou, Fujian, PR China \\ ${ }^{2}$ Division of Oncological Surgery, Fujian Provincial Cancer Hospital, Affiliated Hospital \\ of Fujian Medical University, Fuzhou, Fujian, PR China \\ ${ }^{3}$ Department of Pathology, The First Affiliated Hospital of Fujian Medical University, \\ PR China \\ These two authors contributed equally to this paper.
}

\section{Introduction}

Neuroendocrine carcinomas (NEC) is defined as a high-grade neuroendocrine neoplasm and regarded as a distinct and independent category because of its particularly aggressive biological behaviour and poor prognosis, compared with other neuroendocrine neoplasms such as neuroendocrine tumour/carcinoid [1-5].

Extra-pulmonary NECs are most often found in the gastrointestinal (GI) tract, which accounts for $35-55 \%$ of all extra-pulmonary NECs [6]. GI-NECs are mainly located in the oesophagus, stomach, pancreas, and colon. Among which, gastric NEC (G-NEC) is a rare but highly malignant tumour [7, 8], and it has a poorer outcome in comparison to conventional adenocarcinomas [9-11]. However, because of its rarity, limited study had been directed towards its clinicopathological characteristics and prognosis, and the optimal clinical management strategies have not yet been well established.

G-NECs encompass two histopathological entities: small-cell NECs (SCNECS) and large-cell NECS (LC-NECS). It has been reported that most NECS arising in the glandular mucosa of the gastrointestinal tract are large-cell type, whereas those arising in the squamous mucosa are mostly small-cell type [12], and thus the majority of the NECs are LC-NECs. Both cell types have a high proliferation rate with a Ki-67 labelling index $>20 \%$ by definition, but usually much higher (> 75\%) [13]. They are also characterised by some specific molecular markers of neuroendocrine differentiation. Numerous studies have supported the validity of the Ki-67 labelling index as a prognostic indicator for GI-NETs, as well as its potential role in planning therapy [14-16]. In addition, synaptophysin (Syn) is usually positive, while chromogranin A (CgA) is less frequently present. Positive CgA staining usually indicates a more mature tumour, and the presence of both Syn and CgA is considered a good prognostic sign [17-19].

In order to identify and better define the useful prognostic markers for G-NECs, in the present study, we examined the morphologic, immunohistochemical, as well as clinicopathological factors in 62 cases of G-NEC that met the definition according to the updated World Health Organization (WHO) classification [20].

\section{Material and methods}

Patients and tissue preparation

Sixty-two G-NEC patients were identified among 8052 cases of gastric carcinoma who underwent radical resection of primary tumour (total or 
Table 1. Clinicopathological parameters

\begin{tabular}{|c|c|}
\hline Parameters & No. of cases (\%) \\
\hline Median age, years & 63.4 \\
\hline $\begin{array}{l}\text { Age (y.o.) } \\
\quad<63 \\
\geq 63\end{array}$ & $\begin{array}{l}30(48.4) \\
32(51.6)\end{array}$ \\
\hline $\begin{array}{l}\text { Gender } \\
\text { Male } \\
\text { Female }\end{array}$ & $\begin{array}{l}48(77.4) \\
14(22.6)\end{array}$ \\
\hline $\begin{array}{l}\text { Location of disease } \\
\text { Upper third } \\
\text { Middle third } \\
\text { Lower third }\end{array}$ & $\begin{array}{l}31(50) \\
20(32.3) \\
11(17.7)\end{array}$ \\
\hline $\begin{array}{l}\text { Greatest dimension } \\
\quad<5 \mathrm{~cm} \\
\geq 5 \mathrm{~cm}\end{array}$ & $\begin{array}{l}38(61.3) \\
24(38.7)\end{array}$ \\
\hline $\begin{array}{l}\text { TNM stage } \\
\text { I } \\
\text { II } \\
\text { III } \\
\text { IV }\end{array}$ & $\begin{array}{c}0 \\
6(9.7) \\
48(77.4) \\
8(12.9)\end{array}$ \\
\hline $\begin{array}{l}\text { Histology } \\
\text { Large cell } \\
\text { Small cell }\end{array}$ & $\begin{array}{c}53(85.5) \\
9(14.5)\end{array}$ \\
\hline $\begin{array}{l}\text { Depth of invasion } \\
\text { T1 } \\
\text { T2 } \\
\text { T3 } \\
\text { T4 }\end{array}$ & $\begin{array}{c}1(1.6) \\
6(9.7) \\
26(41.9) \\
29(46.8)\end{array}$ \\
\hline $\begin{array}{l}\text { LV factor } \\
\text { Positive } \\
\text { Negative }\end{array}$ & $\begin{array}{l}49(79.0) \\
13(21.0)\end{array}$ \\
\hline $\begin{array}{l}\text { V-factor } \\
\text { Positive } \\
\text { Negative }\end{array}$ & $\begin{array}{l}37(59.7) \\
25(40.3)\end{array}$ \\
\hline $\begin{array}{l}\text { LN metastasis } \\
\text { Positive } \\
\text { Negative }\end{array}$ & $\begin{array}{l}49(79.0) \\
13(21.0)\end{array}$ \\
\hline $\begin{array}{l}\text { Distant metastasis } \\
\text { Positive } \\
\text { Negative }\end{array}$ & $\begin{array}{l}24(38.7) \\
38(61.3)\end{array}$ \\
\hline $\begin{array}{l}\text { Postoperative chemotherapy } \\
\text { Yes } \\
\text { No }\end{array}$ & $\begin{array}{l}42(67.7) \\
20(32.3)\end{array}$ \\
\hline $\begin{array}{l}\text { Relapse } \\
\text { Yes } \\
\text { No }\end{array}$ & $\begin{array}{l}32(51.6) \\
30(48.4)\end{array}$ \\
\hline $\begin{array}{l}\text { Residual tumour classificatio } \\
\text { R0 } \\
\text { R1 } \\
\text { R2 }\end{array}$ & $\begin{array}{c}53(85.5) \\
4(6.4) \\
5(8.1)\end{array}$ \\
\hline $\begin{array}{l}\text { Ki-67 expression } \\
\text { High } \\
\text { Low }\end{array}$ & $\begin{array}{l}28(45.2) \\
34(54.8)\end{array}$ \\
\hline $\begin{array}{l}\text { Chromogranin A expression } \\
\text { Positive } \\
\text { Negative }\end{array}$ & $\begin{array}{l}36(58.1) \\
26(41.9)\end{array}$ \\
\hline Median follow-up (months) & 19.3 \\
\hline
\end{tabular}

Clinical data were recorded in accordance with the AJCC TNM staging system for carcinoma of the stomach; $L V$-factor $N$-factor - the status of tumour invasion into lymphatic vessels (LV)/vascular invasion (V); $L N$ - lymph node; $R O$ - no residual tumour/R1 - microscopic residual tumour/R2 - macroscopic residual tumour subtotal gastrectomy) and systematic lymphadenectomy at The First Affiliated Hospital of Fujian Medical University (Fuzhou, Fujian Province, P.R.C.) between January 2007 and February 2013. The patients' clinicopathological data are shown in detail in Table 1 . None of the patients had received chemo- or radiotherapy before tissue collection. The histopathological features of the tumour specimens were classified in accordance with the WHO criteria $[20,21]$. The TNM staging was determined according to the $7^{\text {th }}$ Edition of the AJCC guidelines (2010) for NEC. The study protocol was approved by the Human Ethics Review Committee of Fujian Medical University, and signed, informed consent was obtained from each patient. Each specimen was fixed overnight in 10\% buffered formalin at room temperature and embedded in paraffin. Serial sections were cut at a thickness of $4 \mu \mathrm{m}$ and placed onto 3-aminopropyltriethoxysilane-coated glass slides. Some sections were stained with haematoxylin and eosin in a routine manner for histological examination.

\section{Immunohistochemistry for synaptophysin, chromogranin A, and Ki-67}

Immunohistochemistry was performed with the indirect enzyme-labelled antibody method, as described previously $[22,23]$. The antibodies used in IHC are demonstrated in Table 2. In brief, for detection of Ki-67, synaptophysin, and chromogranin A, paraffin-embedded sections were deparaffinised with toluene and rehydrated in graded alcohols. After being autoclaved for 15 minutes at $120^{\circ} \mathrm{C}$ in $10 \mathrm{mM}$ citrate buffer ( $\mathrm{pH}$ 6.0) for antigen retrieval, endogenous peroxidase was inactivated with $0.3 \%$ hydrogen peroxide in methanol for 15 minutes. The sections were then pre-incubated with $500 \mu \mathrm{g} / \mathrm{ml}$ normal goat lgG dissolved in 1\% BSA in PBS ( $\mathrm{pH}$ 7.4) for one hour, reacted with primary antibodies for 16 hours, washed with $0.075 \%$ Brij 35 in PBS, and then incubated with HRP-conjugated goat anti-rabbit IgG (CgA/Syn) or HRP-conjugated goat anti-mouse IgG (Ki-67) in $1 \%$ BSA in PBS for one hour. After washing with $0.075 \%$ Brij 35 in PBS, the sites of HRP were visualised with DAB and $\mathrm{H}_{2} \mathrm{O}_{2}$. Finally, the nuclei were counterstained with haematoxylin. As a negative control, some sections were reacted with normal rabbit IgG or normal mouse IgG instead of the specific antibodies.

\section{Quantitative evaluation}

Staining results were examined by two observers unaware of patients' clinical information. Another reading by a third observer was needed to reach a consensus when there was a significant discrepancy between initial results. At least five high-power fields and more than 2000 cells were calculated in each case with a light microscope (Zeiss 2021-85; Carl Zeiss Inc, Germany) at 400× magnification. Immunostaining results were evaluated by calculating the percentage of positive staining cells.

\section{Statistical analysis}

The SPSS 19.0 statistical software package (SPSS Inc., Chicago, IL, USA) was employed for all analyses. The association between tested markers and different clinicopath- 
Table 2. List of antibodies used in immunohistochemistry

\begin{tabular}{lcc} 
Antibody & Working dilution/concentration & \multicolumn{1}{c}{ Manufacturer } \\
$\begin{array}{l}\text { Monoclonal, mouse anti-human Ki-67 (Clone: MIB-1) } \\
\begin{array}{l}\text { Monoclonal, rabbit anti-human Chromogranin A } \\
\text { (Clone: SP12) }\end{array}\end{array}$ 1:400 & DakoCytomation, Glostrup, Denmark \\
$\begin{array}{l}\text { Monoclonal, rabbit anti-human Synaptophysin } \\
\text { (Clone: EP158) }\end{array}$ & $1: 200$ & Thermo Fisher Scientific, Fremont, \\
Normal goat IgG & $1: 20$ & Epitomics, Inc. Burlingame, CA, USA \\
HRP-conjugated goat anti-rabbit/mouse IgG & $1: 200$ & Sigma Chemical Co., MO, USA
\end{tabular}

ological characteristics of the patients, including age, gender, location, greatest dimension, TNM stage, histology, depth of invasion, lymphatic vessel involvement (LV-factor), vascular invasion ( $V$-factor), lymph node (LN) metastasis, distant metastasis, postoperative chemotherapy, relapse, residual tumour classification, Ki-67 expression, and chromogranin A expression, were evaluated by Pearson's $\chi^{2}$ or Fisher's exact test as appropriate. The Kaplan-Meier method with log-rank test was used to estimate the probability of overall survival. The Cox proportional hazard model was used to evaluate the association between various markers and patient survival. A $p$-value less than 0.05 was considered statistically significant.

\section{Results}

\section{Patients' clinicopathological data}

As shown in Table 1, the patient population included 48 males and 14 females, and the male-to-female ratio was approximately $3: 1$. The mean age of the patients was 63.4 years, ranging from 47 to 82 years. The number of upper, middle, and lower third disease were 31, 20, and 11 , respectively. Invasion into mucosa/submucosa was observed only in one patient, invasion into lamina muscularis propria occurred in six patients, invasion into subserosal connective tissue without involving visceral peritoneum or adjacent structures was observed in 26 cases, and invasion into serosa or adjacent structures occurred in $29 \mathrm{pa}$ tients. There were 24 cases with a greatest dimension of $5 \mathrm{~cm}$ or more, while 38 cases had less than $5 \mathrm{~cm}$. As for TNM staging, six cases were stage II, 48 cases stage III, and 8 cases were stage IV. Forty-nine patients were present with LN metastasis while 13 were absent. Twenty-four patients were found positive with distant metastasis while 38 negative. All patients underwent surgical resection of the stomach with regional lymphadenectomy, and 53 cases underwent curative resection, i.e. R0 resection, while four were R1 resection, and the other five were R2 resection. In detail, 11 underwent distal gastrectomy and 51 total gastrectomy. Combined resection of spleen and tail of pancreas was performed in eight patients with advanced tumours in the upper third stomach. Resection was aggressively extended to adjacent organs when tumour invasion was suspected, including liver $(n=5)$ and transverse colon $(n=3)$. Nonetheless, macroscopic tumour residual was found in five patients and microscopic tumour residual in four cases. Forty-two patients received postoperative adjuvant chemotherapy and 20 did not. The average num- ber of adjuvant chemotherapy cycle was four. Regimens of adjuvant chemotherapy were as below: fluorouracil, leucovorin plus oxaliplatin (FOLFOX4) for 13 patients, fluconazole plus oxaliplatin for 12 patients, paclitaxel plus oxaliplatin for four patients, docetaxel plus fluconazole for four patients, dacarbazine, epirubicin plus fluorouracil for two patients, and other regimens for the other seven patients. Thirty-two patients relapsed after surgery, while no sign of relapse had been found at the end-point of follow-up for the other 30 cases. Fifty-three cases were large-cell NECs and nine were small-cell NECs. Postoperative follow-up data were available for all 62 cases, and the median follow-up duration was 19.3 months, ranging from 1.0 to 54.0 months.

\section{Histological findings and immunohistochemical staining for synaptophysin, chromogranin A, and $\mathrm{Ki}-67$}

The tumors were sub-classified into large-cell and smallcell NECs, as defined by the WHO classification and previous reports [4, 24]. LC-NECs manifested as tumour cells exhibiting round to markedly irregular nuclei with coarse chromatin and prominent nucleoli and moderate amounts of cytoplasm (Fig. 1A). Peripheral palisading and/or rosette formation were present in the majority of LC-NECs, which also exhibited hyperchromatic nuclei with finely granular chromatin and eosinophilic granular cytoplasm. SC-NECS, however, showed a markedly higher nuclear/cytoplasmic ratio, exhibiting hyperchromatic nuclei with finely granular chromatin and scant cytoplasm (Fig. 1B). Other factors like presence of necrosis, mitotic counts (count per $10 \mathrm{HPF}$ using a Zeiss 2021-85 microscope), lymphatic and vascular involvement, association with non-NEC components, and the statuses of the surrounding mucosa, including the presence of chronic gastritis and intestinal metaplasia, were also evaluated.

Immunohistochemically, Ki-67 was localised predominantly in the nuclei, while both chromogranin A and synaptophysin in the cytoplasm (Fig. 1G-H). Here we used $56 \%$ as the cut-off point to determine high and low Ki-67 expression. The number of patients positive for synaptophysin staining was 61 (98.4\%), whereas the numbers for positive chromogranin A staining and high Ki-67 expression was 26 (41.9\%) and 28 (45.2\%), respectively. For different histologies, 25 of 53 LC-NECs were found to have high expression of Ki-67 while in SC-NECs the number was three $(p=0.494)$. As for chromogranin A expression, 27 of 53 large-cell and all nine small-cell NECs were positive 

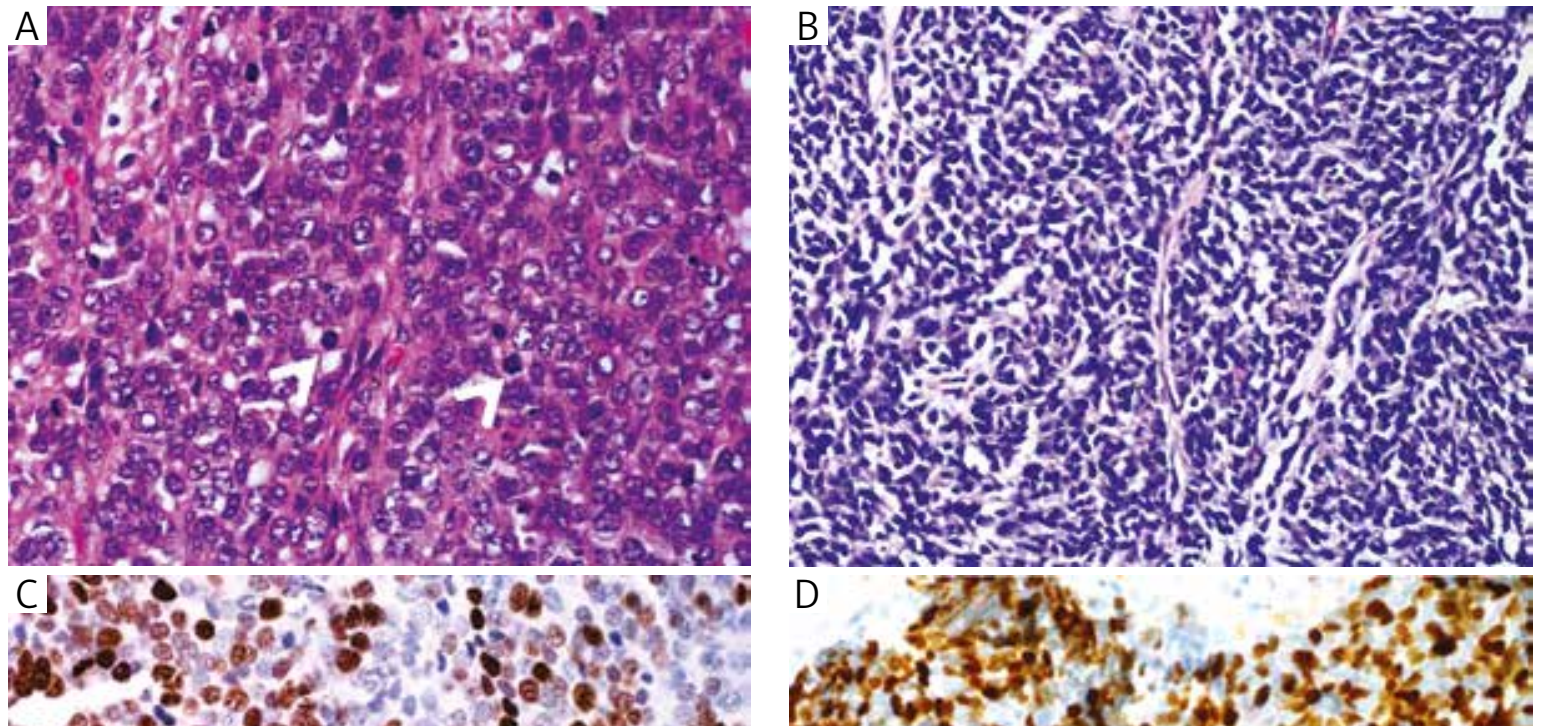

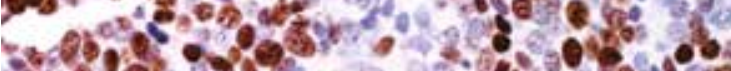

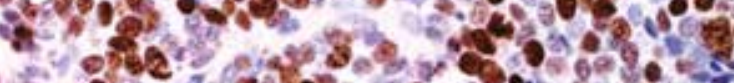

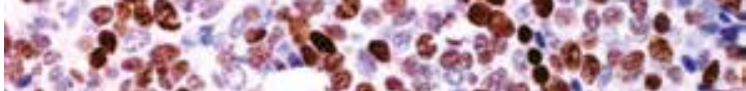

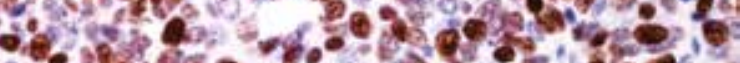
ie. भes. 8 and of

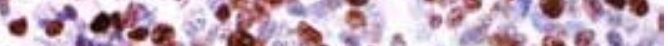

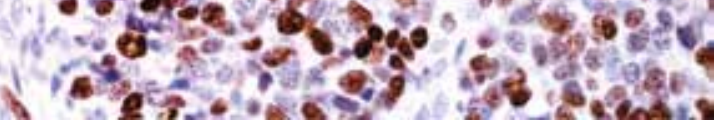
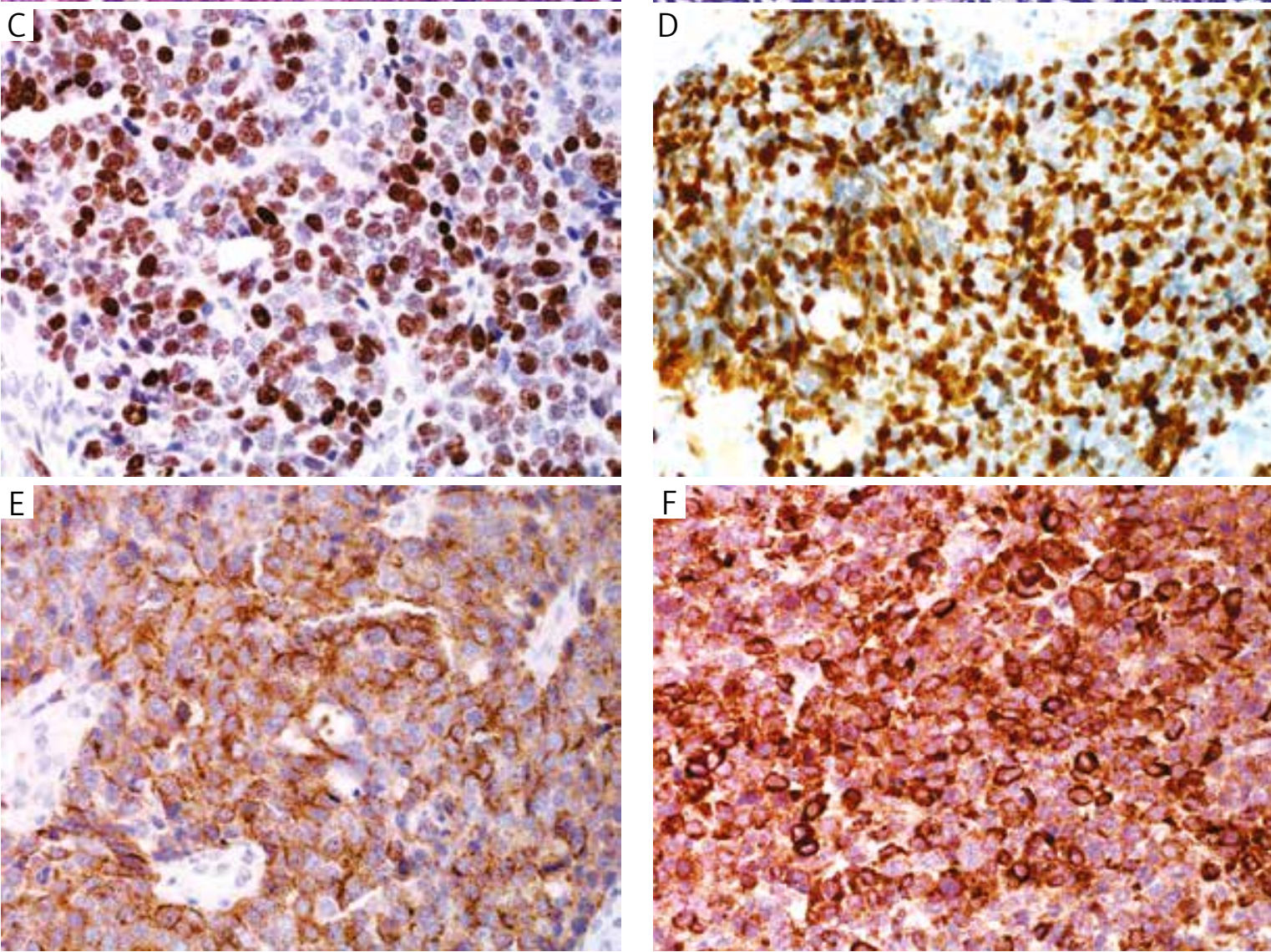

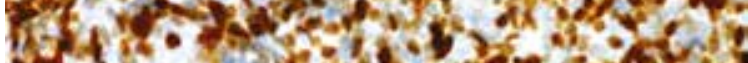
I.

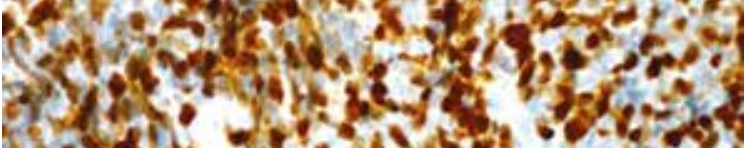

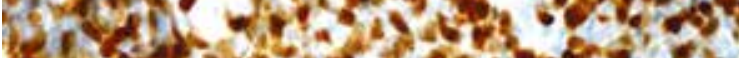

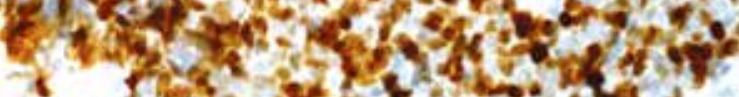
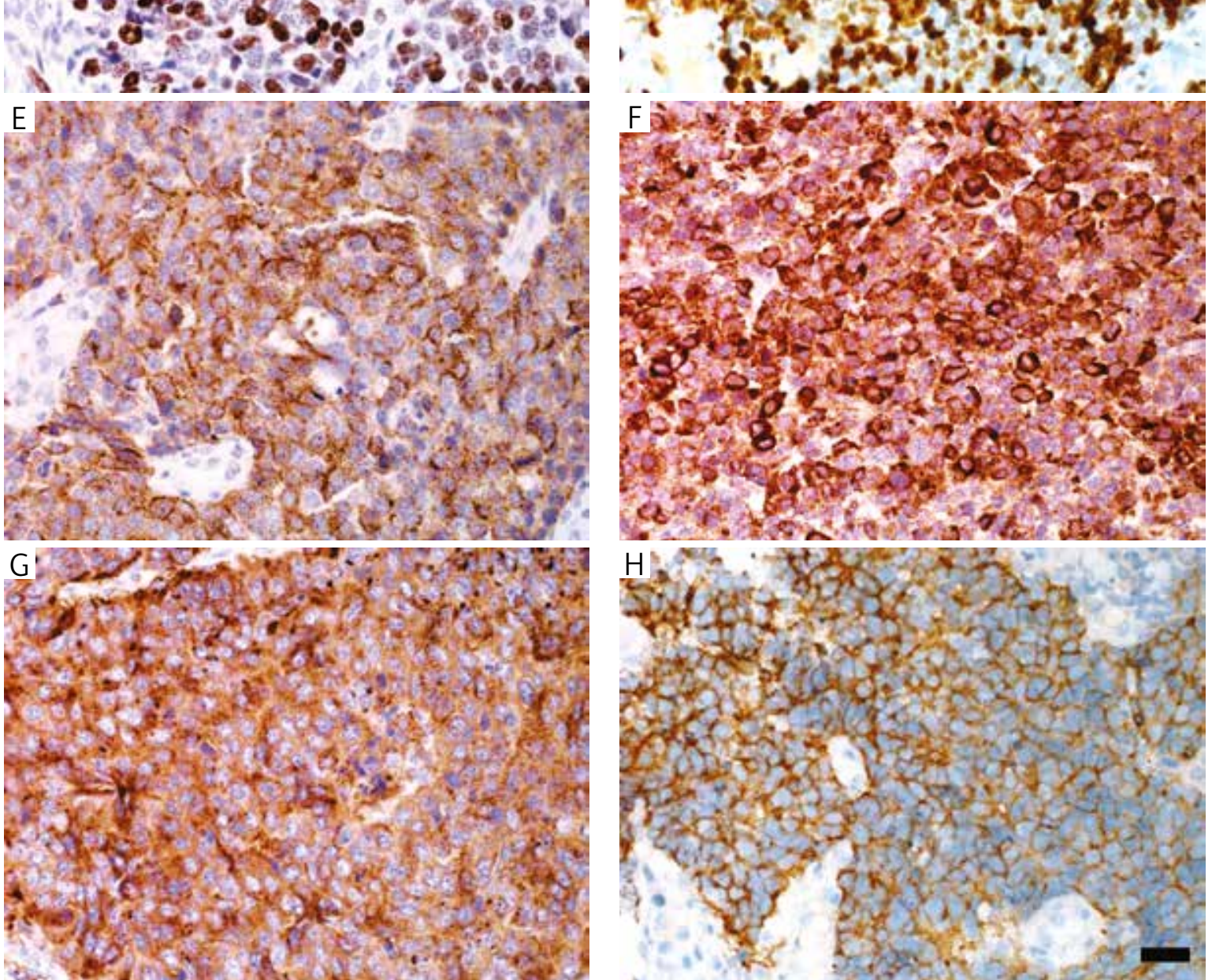

Fig. 1. Histologic and immunohistochemical features of LC-NEC and SC-NEC. A) HE staining for large-cell NEC with a trabecular growth pattern. Of note, the tumour cells have round and hyperchromatic nuclei and eosinophilic and granular cytoplasm (white arrowheads), showing nuclear moulding and suggesting neuroendocrine differentiation; B) HE staining for small-cell NEC, showing a markedly higher nuclear/cytoplasmic ratio and hyperchromatic nuclei with finely granular chromatin and scant cytoplasm; C) LC-NEC, positive staining for Ki-67; D) SC-NEC, positive staining for Ki-67; E) LC-NEC, positive staining for chromogranin A; F) SC-NEC, positive staining for chromogranin A; G) LC-NEC, positive staining for synaptophysin; H) SC-NEC, positive staining for synaptophysin. Scale bar represents $20 \mu \mathrm{m}$ 
Table 3. Association of Ki-67 and chromogranin A with clinicopathological parameters in G-NEC patients

\begin{tabular}{|c|c|c|c|c|c|c|c|c|}
\hline \multirow[t]{2}{*}{ Variables } & \multicolumn{4}{|c|}{$\mathrm{Ki}-67$} & \multicolumn{4}{|c|}{ CgA } \\
\hline & All cases & $\mathrm{H}$ & $\mathrm{L}$ & $p$ & All cases & $\mathrm{H}$ & $\mathrm{L}$ & $p$ \\
\hline $\operatorname{Age}^{a}$ (y.o.) & & & & 0.459 & & & & 0.829 \\
\hline$\geq 63$ & 32 & 13 & 19 & & 32 & 19 & 13 & \\
\hline$<63$ & 30 & 15 & 15 & & 30 & 17 & 13 & \\
\hline Gender & & & & 0.420 & & & & 0.937 \\
\hline Male & 48 & 23 & 25 & & 48 & 28 & 20 & \\
\hline Female & 14 & 5 & 9 & & 14 & 8 & 6 & \\
\hline Location & & & & 0.005 & & & & 0.876 \\
\hline Upper & 31 & 9 & 22 & & 31 & 19 & 12 & \\
\hline Middle & 20 & 15 & 5 & & 20 & 11 & 9 & \\
\hline Lower & 11 & 4 & 7 & & 11 & 6 & 5 & \\
\hline TNM stage $^{b}$ & & & & 0.681 & & & & 1.000 \\
\hline |/II & 6 & 2 & 4 & & 6 & 4 & 2 & \\
\hline III/IV & 56 & 26 & 30 & & 56 & 32 & 24 & \\
\hline Histology & & & & 0.494 & & & & 0.008 \\
\hline Small-cell & 9 & 3 & 6 & & 9 & 9 & 0 & \\
\hline Large-cell & 53 & 25 & 28 & & 53 & 27 & 26 & \\
\hline Depth of invasion ${ }^{b}$ & & & & 0.442 & & & & 0.119 \\
\hline T1-2 & 7 & 2 & 5 & & 7 & 2 & 5 & \\
\hline T3-4 & 55 & 26 & 29 & & 55 & 34 & 21 & \\
\hline LV-factor & & & & 0.585 & & & & 0.775 \\
\hline Yes & 49 & 23 & 26 & & 49 & 28 & 21 & \\
\hline No & 13 & 5 & 8 & & 13 & 8 & 5 & \\
\hline$V$-factor & & & & 0.006 & & & & 0.426 \\
\hline Yes & 37 & 22 & 15 & & 37 & 23 & 14 & \\
\hline No & 25 & 6 & 19 & & 25 & 13 & 12 & \\
\hline LN metastasis & & & & 0.585 & & & & 0.775 \\
\hline Yes & 49 & 23 & 26 & & 49 & 28 & 21 & \\
\hline No & 13 & 5 & 8 & & 13 & 8 & 5 & \\
\hline Distant metastasis & & & & 0.660 & & & & 0.574 \\
\hline Yes & 24 & 10 & 14 & & 24 & 15 & 9 & \\
\hline No & 38 & 18 & 20 & & 38 & 21 & 17 & \\
\hline Greatest dimension & & & & 0.257 & & & & 0.038 \\
\hline$\geq 5 \mathrm{~cm}$ & 24 & 13 & 11 & & 24 & 10 & 14 & \\
\hline$<5 \mathrm{~cm}$ & 38 & 15 & 23 & & 38 & 26 & 12 & \\
\hline Relapse & & & & 0.078 & & & & 0.829 \\
\hline Yes & 32 & 11 & 21 & & 32 & 19 & 13 & \\
\hline No & 30 & 17 & 13 & & 30 & 17 & 13 & \\
\hline Ki-67 & - & - & - & - & & & & 0.243 \\
\hline High & & & & & 28 & 14 & 14 & \\
\hline Low & & & & & 34 & 22 & 12 & \\
\hline
\end{tabular}

$L V$-factor-lymphatic vessel involvement; $V$-factor - vascular involvement; LN metastasis - lymph node metastasis

a Mean age

${ }^{b}$ Fisher exact tests (2-sided); $\chi^{2}$ test for all the other analyses.

( $p=0.008)$. No statistical significance was found for synaptophysin expression within two NECs $(p>0.1)$.

\section{Correlation of Ki-67and chromogranin A expression with clinicopathological parameters}

To determine the correlation of Ki-67 and chromogranin A expression and clinicopathological parameters, and to determine its prognostic impact, chi-square together with univariate, multivariate, and Kaplan-Meier survival analyses were performed. As shown in Table 3, high Ki-67 expression was significantly associated with the mid-third disease $(p=0.005)$, vascular involvement $(p=0.006)$, and had a trend of significant correlation with tumour relapse $(p=0.078)$. High expression of chromogranin A was significantly associated with small-cell histology $(p=0.008)$ and lesser greatest dimension of tumour $(\leq 5 \mathrm{~cm}, p=$ 0.038). However, Kaplan-Meier survival analyses revealed no difference of disease-free survival or OS in patients with different levels of Ki-67 or chromogranin A expression $(p>0.05)$. Univariate analysis demonstrated associations between poor prognosis and several factors, including high TNM staging $(p=0.048)$, vascular involvement ( $p=$ $0.023)$, relapse $(p=0.004)$, and microscopic/macroscopic residual tumour (R1/2, $p<0.001)$. Using a Cox proportional 
Table 4. Univariate and multivariate analyses of factors associated with O.S.

\begin{tabular}{|c|c|c|}
\hline Variables & Hazard Ratio $(95 \% \mathrm{Cl})$ & $P$-value \\
\hline \multicolumn{3}{|l|}{ Univariate analysis } \\
\hline Age (y.o.) ( $\geq 63$ vs. < 63) & $1.519(0.735-3.140)$ & 0.260 \\
\hline Gender (male vs. female) & $1.688(0.645-4.419)$ & 0.286 \\
\hline Location (upper/middle/lower) & $1.139(0.730-1.778)$ & 0.566 \\
\hline $\begin{array}{l}\text { Greatest dimension }(\mathrm{cm}) \\
(\geq 5 \text { vs. }<5)\end{array}$ & $0.594(0.290-1.219)$ & 0.156 \\
\hline TNM stage (I/II vs. III/IV) & $7.587(1.019-56.464)$ & 0.048 \\
\hline $\begin{array}{l}\text { Histology (small cell vs. } \\
\text { large cell) }\end{array}$ & $0.700(0.243-2.020)$ & 0.510 \\
\hline Depth of invasion(T1-2 vs. T3-4) & $1.028(0.357-2.957)$ & 0.959 \\
\hline LV-factor (yes vs. no) & $1.894(0.754-4.757)$ & 0.174 \\
\hline V-factor (yes vs. no) & $2.508(1.137-5.533)$ & 0.023 \\
\hline LN metastasis (yes vs. no) & $1.894(0.754-4.757)$ & 0.174 \\
\hline Distant metastasis (yes vs. no) & $1.654(0.807-3.390)$ & 0.169 \\
\hline Relapse (yes vs. no) & 77.455 (4.036-1486.380) & 0.004 \\
\hline $\begin{array}{l}\text { Residual tumour classification } \\
\text { (RO vs. R1/2) }\end{array}$ & $5.911(2.544-13.735)$ & 0.000 \\
\hline Ki-67 expression (high vs. low) & $0.635(0.297-1.359)$ & 0.242 \\
\hline $\begin{array}{l}\text { Chromogranin A } \\
\text { (positive vs. negative) }\end{array}$ & $0.898(0.435-1.851)$ & 0.770 \\
\hline \multicolumn{3}{|l|}{ Multivariate analysis } \\
\hline Relapse (yes vs. no) & 77.455 (4.036-1486.380) & 0.004 \\
\hline
\end{tabular}

hazard regression analysis, we found that relapse was the one and only independent predictor associated with poor prognostic outcome (Table 4).

\section{Survival and its relation with clinicopathological factors}

As shown in Figure 2B-F, median OS was 28 months. For histology of large- and small-cell NECs, their median survival was 29 and 28 months, respectively ( $p=0.3964)$. Furthermore, for stage III the median survival was 26 months, and 11 months for stage IV ( $p=0.0033)$. Owing to the limited number of stage II cases, its median survival could not be determined. In addition, for 42 patients given postoperative chemotherapy their median survival was 29 months, while it was 22 months for 20 patients without postoperative chemotherapy $(p=0.0303)$. For patients with different level of Ki-67 expression, the median survival was 26 and 30 months, respectively ( $p=0.2287$ ).

As shown in Table 4, univariate analysis revealed that TNM staging (I/II vs. III/IV; $p=0.048$ ), angioinvasion ( $\mathrm{V}$-factor, yes vs. no; $p=0.023$ ), tumour relapse (yes vs. no; $p=0.004$ ), and residual tumour classification (RO vs. R1/2; $p<0.001$ ) were significantly correlated with survival (tumour depth, lymph node metastasis, and residual tumour classification/curability were classified according to the seventh edition of AJCC for G-NECS). Multivariate analysis indicated that tumour relapse (hazard ratio, 77.455; 95\% confidence interval, 4.036-1486.380; $p=0.004$ ) was the sole independent prognostic factor for survival.

\section{Discussion}

In the present study, we investigated the expressions of Ki-67, chromogranin A, and synaptophysin in G-NECs immunohistochemically, analysed the associations between the tested markers and clinicopathological parameters, and tried to determine the prognostic factors that would probably affect the survival.

First of all, we found that high expression of Ki-67 was significantly associated with middle third disease of the stomach $(p=0.005)$ and vascular infiltration $(p=0.006)$, and had a trend of significant correlation with tumour relapse ( $p=0.078)$. Ki-67 was reported to be overexpressed at both mRNA and protein levels in many cancerous tissues [25-28], and correlated with invasiveness, increased proliferation, and poor outcome. Sorbye et al. [29] reported that patients with a Ki-67 less than 55\% were less responsive to platinum-based chemotherapy, but had a longer survival. Boo et al. [13] demonstrated that a high Ki-67 PI (>60\%) was correlated with tumour recurrence and worse histological differentiation. Boo et al. [13] used $60 \%$ without any specific explanation, while Sorbye et al. [29] used $55 \%$ as the cutoff point determined by receiver operating characteristic (ROC) analysis, which sounded more reasonable. In the present study, we used $56 \%$ as the cut-off point, and found that the high Ki-67 PI was closely related to middle third disease of the stomach $(p=0.005)$ and vascular infiltration $(p=0.006)$, and had a trend of significant correlation with tumour relapse $(p=0.078)$, which was consistent with the findings of Rindi et al. [30]. To our knowledge, our data demonstrated for the first time a direct association of expression of $\mathrm{Ki}-67$ with vascular invasion and location of disease of G-NECs. Although in our study neither univariate $(p=0.242)$ nor multivariate analysis revealed high $\mathrm{Ki}-67 \mathrm{PI}$ as a poor prognostic factor in G-NECs, the result still suggested that high proliferation index contributed to worse prognosis because both angioinvasion and relapse were confirmatively affecting the outcome of G-NEC patients.

Chromogranin A (CgA) is localised in neurosecretory granules, and its expression thus reflects the degree of neuroendocrine differentiation. According to Bakkelund's findings [31], signet ring cells in gastric carcinomas were derived from neuroendocrine cells. Fujiyoshi et al. [17] demonstrated that a significant positive correlation existed between CgA positivity and favourable prognosis, with a tendency for greater positivity to be associated with better OS. In the current study, our finding indicated that high expression of chromogranin A was significantly associated with various clinicopathological parameters including small-cell histology of NECs $(p=0.008)$ and lesser tumour greatest dimension $(p=0.038)$, which were consistent with the findings of Pape et al. [18] and Welin et al. [19], while we failed to confirm the significant correlation with better prognosis $(p=0.770)$. Further study with a large scale of specimens would be needed.

Most significantly, we found that high TNM staging ( $p=0.048)$, vascular involvement $(p=0.023)$, relapse ( $p=0.004)$, and microscopic/macroscopic residual tumour $(\mathrm{R} 1 / 2, p<0.001)$ were closely associated with poor out- 

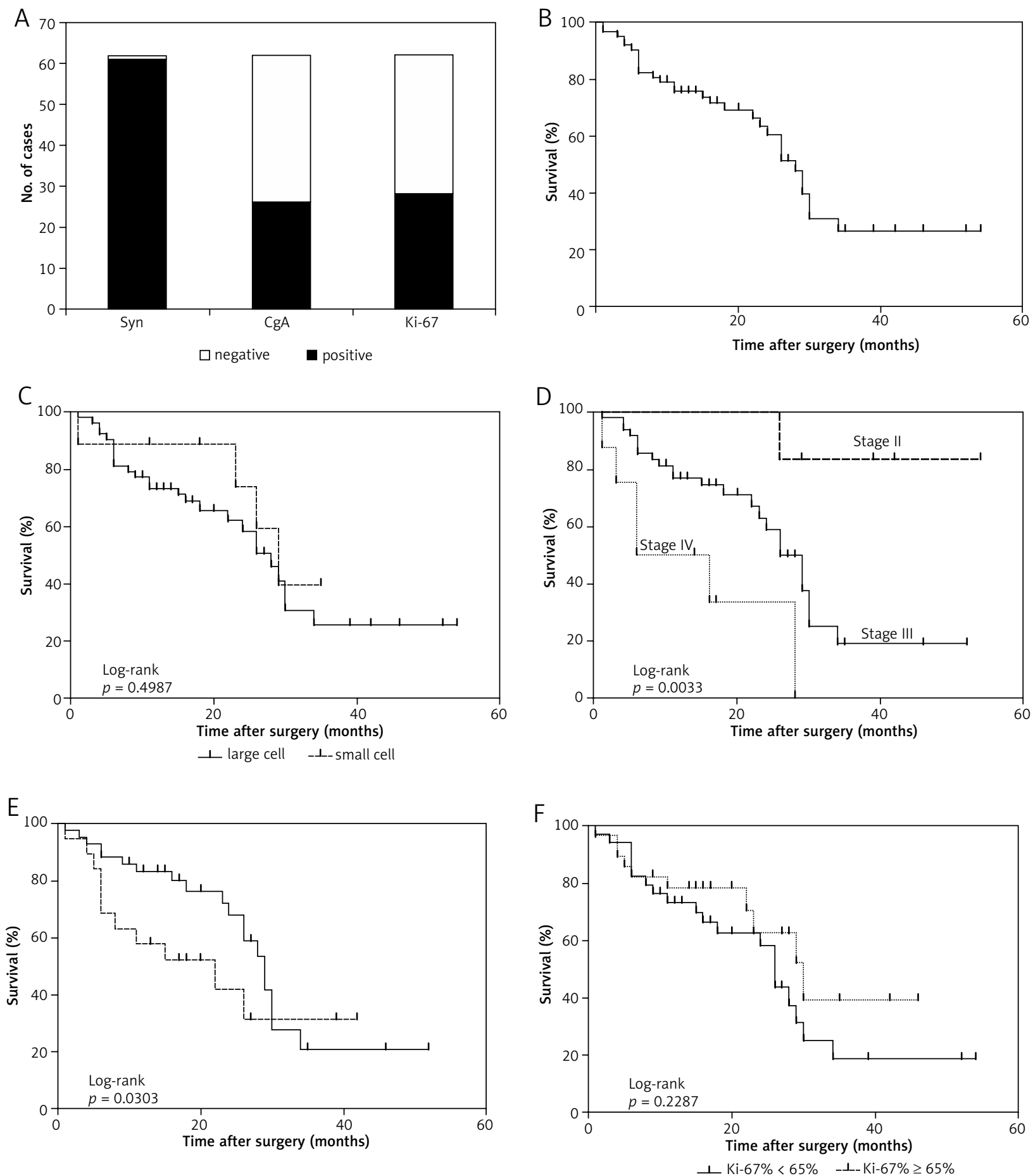

Fig. 2. Kaplan-Meier cumulative survival curves and log-rank test. A) Chart illustration of number of cases of immunohistochemical staining for synaptophysin, chromogranin A, and Ki-67; B) Overall survival; C) large-cell NECs versus small-cell NECs ( $p=0.4987)$; D) Survival according to AJCC stages, $7^{\text {th }}$ Edition ( $\left.p=0.0033\right)$; E) Presence and absence of postoperative adjuvant chemotherapy $(p=0.0303)$; F) high Ki-67 expression vs. low Ki-67 expression $(p=0.2287)$

come (Table 4). In general, TNM staging, vascular involvement, tumour relapse and residual tumour classification were considered the most important factors affecting prognosis in GI-NECs [11,30], as was also confirmed in the present study. In patients with $\mathrm{R} 1 / 2$ resection, their hazard ratio of tumour recurrence was 4.911-fold higher than those with $\mathrm{RO}$ resection (Table 4). Kubota et al. [32] reported maximum tumour diameter, depth of tumour invasion,
LN metastasis, lymphatic invasion, p-Stage, and curability had significant correlations with survival. Recently, Ishida et al. [11] confirmed curative surgery as the only independent prognostic factor related to survival. Although our findings demonstrated that surgical curability is one of the prognostic factors of vital importance, which would inevitably affect the probability of relapse and long-term outcome, it is not confirmed as an independent one. How- 
ever, our data suggested relapse as the only independent prognostic factor.

When further investigating the factors resulting in unfavourable outcome, we inferred that poor prognosis seemed to result from potent microvessel invasiveness and subsequent frequent further metastasis to lymph nodes or other organs even in the early stages of the disease [3], and a significant correlation between liver metastasis and vascular invasion has been well demonstrated in gastric cancers [33-35]. Chiba et al. [36] revealed in their retrospective review of 119 cases that G-NECs seemed significantly to have more frequent incidences of lymphatic invasion (88.9\% vs. 56.6\%), vascular invasion (75.6\% vs. $31.6 \%$ ), and LN metastases (82.1\% vs. 58.8\%) compared to those in ordinary gastric cancer. In our study, lymphatic invasion and LN metastasis were present in 49 cases (79.0\%), and vascular invasion in 37 cases (59.7\%). Furthermore, univariate analysis suggested the importance of vascular invasion ( $p=0.023)$ and curability $(p<0.001)$ for survival (Table 4). Based on these facts, we suggest that every effort should be made to achieve an RO resection for every surgical candidate, so as to obtain a better outcome and postpone the event of tumour relapse.

Our findings also suggested that there was obvious discrepancy with the OS between patients receiving postoperative chemotherapy or not. As shown in Figure 2E, patients who underwent postoperative chemotherapy had a significantly better outcome with a median overall survival of 29 months; much longer than those who did not, which was only 22 months. In our patient cohort, the common regimens used were fluorouracil, leucovorin plus oxaliplatin (FOLFOX4), fluconazole plus oxaliplatin, paclitaxel plus oxaliplatin, and docetaxel plus fluconazole. In addition, Okita et al. [37] reported that chemotherapy with cisplatin plus irinotecan was also effective for gastric poorly-differentiated NECs, whose response rate was 75\%, median progression-free survival time was seven months, and median survival was 22.6 months.

Some limitations of this research should be noted. First of all, the number of the dataset was small, so the statistical power would be somewhat limited. Further study with a larger sample size would be necessary to validate the present results. Secondly, some of the follow-up time of the cases is somewhat short. Although other regimens like radiotherapy would be used after progression had been proven, the contribution of other therapies to overall survival had not been taken into account in the survival analysis because surgery plus chemotherapy was considered the key therapy for patients at this stage, and thus would probably lead to some bias.

In summary, G-NEC patients have a poor prognosis, and surgical candidates should be considered for no-residual resection and postoperative chemotherapy without delay. In addition, TNM staging, vascular involvement, and relapse were the most important negative prognostic factors for survival in this retrospective study. In contrast, despite the significant morphologic diversity of G-NECs, their histologic subclassification was not of prognostic significance.
This study was supported in part by a Grant-in-Aid for Youth Scientific Research from the Fujian Provincial Administration of Health (No. 2013-1-10 to X. Chen) and the young backbone talents training foundation of the Fujian healthcare system (No. 2013-ZQN-JC-9 to M. Ying).

The authors declare no conflict of interest.

\section{References}

1. Kloppel G, Clemens A. The biological relevance of gastric neuroendocrine tumors. Yale J Biol Med 1996; 69: 69-74.

2. Rindi G, Bordi C, Rappel S, La Rosa S, Stolte M, Solcia E. Gastric carcinoids and neuroendocrine carcinomas: pathogenesis, pathology, and behavior. World J Surg 1996; 20: 168-72.

3. Bordi C, Azzoni C, D’Adda T, Caruana P, Carlinfante G. Neuroendocrine tumors of the stomach. Pathogenesis, classification and clinical aspects. Pathologe 1997; 18: 313-21.

4. Matsui K, Jin XM, Kitagawa M, Miwa A. Clinicopathologic features of neuroendocrine carcinomas of the stomach: appraisal of small cell and large cell variants. Arch Pathol Lab Med 1998; 122: 1010-7.

5. Waldum HL, Aase S, Kvetnoi I, et al. Neuroendocrine differentiation in human gastric carcinoma. Cancer 1998; 83: 435-44.

6. Walenkamp AM, Sonke GS, Sleijfer DT. Clinical and therapeutic aspects of extrapulmonary small cell carcinoma. Cancer Treat Rev 2009; 35: 228-36.

7. Rindi G, Luinetti O, Cornaggia M, Capella C, Solcia E. Three subtypes of gastric argyrophil carcinoid and the gastric neuroendocrine carcinoma: a clinicopathologic study. Gastroenterology 1993; 104: 994-1006.

8. Bordi C. Endocrine tumours of the stomach. Pathol Res Pract 1995; 191: 373-80.

9. Namikawa T, Oki T, Kitagawa H, Okabayashi T, Kobayashi M, Hanazaki K. Neuroendocrine carcinoma of the stomach: clinicopathological and immunohistochemical evaluation. Med Mol Morphol 2013; 46: 34-40.

10. Louthan O. Neuroendocrine neoplasms of the stomach. Biomed Pap Med Fac Univ Palacky Olomouc Czech Repub 2014; 158: 45560.

11. Ishida M, Sekine S, Fukagawa T, et al. Neuroendocrine carcinoma of the stomach: morphologic and immunohistochemical characteristics and prognosis. Am J Surg Pathol 2013; 37: 949-59.

12. Shia J, Tang LH, Weiser MR, et al. Is nonsmall cell type high-grade neuroendocrine carcinoma of the tubular gastrointestinal tract a distinct disease entity? Am J Surg Pathol 2008; 32: 719-31.

13. Boo YJ, Park SS, Kim JH, Mok YJ, Kim SJ, Kim CS. Gastric neuroendocrine carcinoma: clinicopathologic review and immunohistochemical study of E-cadherin and Ki-67 as prognostic markers. J Surg Oncol 2007; 95: 110-7.

14. Rorstad O. Prognostic indicators for carcinoid neuroendocrine tumors of the gastrointestinal tract. J Surg Oncol 2005; 89: 151-60.

15. Kawahara M, Kammori M, Kanauchi H, Noguchi C, Kuramoto S, Kaminishi M, Endo H, Takubo K. Immunohistochemical prognostic indicators of gastrointestinal carcinoid tumours. Eur J Surg Oncol 2002; 28: 140-6.

16. Criscitiello C, Disalvatore D, De Laurentiis M, et al. High Ki-67 score is indicative of a greater benefit from adjuvant chemotherapy when added to endocrine therapy in Luminal B HER2 negative and node-positive breast cancer. Breast 2014; 23: 69-75.

17. Fujiyoshi Y, Eimoto T. Chromogranin A expression correlates with tumour cell type and prognosis in signet ring cell carcinoma of the stomach. Histopathology 2008; 52: 305-13.

18. Pape UF, Berndt U, Muller-Nordhorn J, Bohmig M, Roll S, Koch M, Willich SN, Wiedenmann B. Prognostic factors of long-term outcome in gastroenteropancreatic neuroendocrine tumours. Endocr Relat Cancer 2008; 15: 1083-97.

19. Welin S, Sorbye H, Sebjornsen S, Knappskog S, Busch C, Oberg K. Clinical effect of temozolomide-based chemotherapy in poorly 
differentiated endocrine carcinoma after progression on first-line chemotherapy. Cancer 2011; 117: 4617-22.

20. Rindi G. The ENETS guidelines: the new TNM classification system. Tumori 2010; 96: 806-9.

21. Rindi G, Inzani F, Solcia E. Pathology of gastrointestinal disorders. Endocrinol Metab Clin North Am 2010; 39: 713-27.

22. Chen X, Song N, Matsumoto K, et al. High expression of trimethylated histone $\mathrm{H} 3$ at lysine 27 predicts better prognosis in non-small cell lung cancer. Int J Oncol 2013; 43: 1467-80.

23. Song N, Liu J, An S, Nishino T, Hishikawa Y, Koji T. (2011). Immunohistochemical analysis of histone $\mathrm{H} 3$ modifications in germ cells during mouse spermatogenesis. Acta Histochem Cytochem 2011; 44: 183-90.

24. Jiang SX, Mikami T, Umezawa A, Saegusa M, Kameya T, Okayasu I. Gastric large cell neuroendocrine carcinomas: a distinct clinicopathologic entity. Am J Surg Pathol 2006; 30: 945-53.

25. Re M, Zizzi A, Ferrante L, et al. p63 and Ki-67 immunostainings in laryngeal squamous cell carcinoma are related to survival. Eur Arch Otorhinolaryngol 2014; 271: 1641-51.

26. Pavelin S, Becic K, Forempoher G, et al. The significance of immunohistochemical expression of Merlin, Ki-67, and p53 in meningiomas. Appl Immunohistochem Mol Morphol 2014; 22: 46-9.

27. Nishimiya H, Kosaka Y, Yamashita K, et al. Prognostic significance of Ki-67 in chemotherapy-naive breast cancer patients with 10year follow-up. Anticancer Res 2014; 34: 259-68.

28. Krabbe LM, Bagrodia A, Lotan Y, et al. Prospective analysis of ki67 as an independent predictor of oncologic outcomes in patients with high grade upper tract urothelial carcinoma. J Urol 2014; 191: 28-34.

29. Sorbye $\mathrm{H}$, Welin S, Langer SW, et al. Predictive and prognostic factors for treatment and survival in 305 patients with advanced gastrointestinal neuroendocrine carcinoma (WHO G3): the NORDIC NEC study. Ann Oncol 2013; 24: 152-60.

30. Rindi G, Azzoni C, La Rosa S, et al. ECL cell tumor and poorly differentiated endocrine carcinoma of the stomach: prognostic evaluation by pathological analysis. Gastroenterology 1999; 116: 532-42.

31. Bakkelund K, Fossmark R, Nordrum I, Waldum H. Signet ring cells in gastric carcinomas are derived from neuroendocrine cells. J Histochem Cytochem 2006; 54: 615-21.

32. Kubota T, Ohyama S, Hiki N, Nunobe S, Yamamoto N, Yamaguchi T. Endocrine carcinoma of the stomach: clinicopathological analysis of 27 surgically treated cases in a single institute. Gastric Cancer 2012; 15: 323-30.

33. Ikeguchi M, Katano K, Oka A, Tsujitani S, Maeta M, Kaibara N. Relationship between hematogenic metastasis of gastric cancer and the maximum extent of venous invasion by cancer cells in the gastric wall. Hepatogastroenterology 1995; 42: 660-5.

34. Maehara Y, Kabashima A, Koga T, Tokunaga E, Takeuchi H, Kakeji Y, Sugimachi K. Vascular invasion and potential for tumor angiogenesis and metastasis in gastric carcinoma. Surgery 2000; 128 : 408-16.

35. Ishida M, Morita S, Saka M, Fukagawa T, Taniguchi H, Katai H. Metachronous liver metastasis from early gastric cancer. I Gastrointest Surg 2012; 16: 837-41.

36. Chiba N, Suwa T, Hori M, Sakuma M, Kitajima M. Advanced gas tric endocrine cell carcinoma with distant lymph node metastasis: a case report and clinicopathological characteristics of the disease. Gastric Cancer 2004; 7: 122-7.

37. Okita NT, Kato K, Takahari D, et al. Neuroendocrine tumors of the stomach: chemotherapy with cisplatin plus irinotecan is effective for gastric poorly-differentiated neuroendocrine carcinoma. Gastric Cancer 2011; 14: 161-5.

\section{Address for correspondence}

\section{Yujie Deng, MD}

Department of Chemotherapy

The First Affiliated Hospital of Fujian Medical University 20 Chazhong Road Taijiang District

Fuzhou, Fujian 350005, PR China

tel. +86-591-8798-1708

fax: +86-591-8639-7967

e-mail: yujiedeng1984@hotmail.com

Submitted: 4.11 .2014

Accepted: $\quad 7.04 .2015$ 